\title{
Grobgeschiebestatistik als Hilfsmittel bei der Kartierung eiszeitlicher Halte
}

\author{
Von Ernst Th. Seraphim, Sennestadt
}

\author{
Mit 1 Abbildung
}

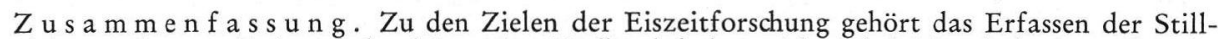
standslagen des nordischen Inlandeises. Im Tiefland haben sich dabei Morphologie und Leitgeschiebestatistik bewährt. In mittelgebirgigen Landschaften setzen erosive Abtragung der Moränen und fluviatile Umlagerung von Geschieben der Anwendung dieser Methoden oft enge Grenzen.

Hier bietet sich als zusätzliche Methode die Grobgeschiebestatistik an. Danach lassen sich glaziale Halte auch aus der Ansammlung von fluviatil nicht transportablen groben nordischen Blöcken in schmalen Streifen im Gelände ablesen.

Der Beitrag befaßt sich weiterhin mit den kritischen Einschränkungen des Aussagewertes der Methode, vor allem in Relation zu den herkömmlichen Verfahren. Einige Hinweise sollen der praktischen Arbeit mit der Grobgeschiebestatistik im Gelände dienen.

$\mathrm{S} \mathrm{u} \mathrm{m} \mathrm{mary}$. One of the aims of Ice Age research is the mapping of the still-stand positions of northern inland ice. The characteristics and statistical analysis of Skandinavian indicator boulders have proved their worth in the North German lowlands but over the mountainous terrains (to the south) erosive denudation of the moraines and the redistribution of the boulders by streaming water narrow the use of the method. In this latter case statistical work on particularly large boulders is a useful supplement. The location of still-stand positions can be derived from the concentrations of these erratics in lines on the ground, for they were too large to be moved by flowing water. Critical reservations on the scope of the method are discussed and its use tied in to that of the traditional ways. Some hints are offered on the practice of the method.

Zahl und Verlauf eiszeitlicher Halte gebührt besondere Beachtung, da ihre Kenntnis zur Auflösung des gesamtpleistozänen Geschehens in Unterabschnitte, aber auch zur Beurteilung der Größenordnung dieser Abschnitte wertvolle Beiträge leisten kann. Deshalb ist es ein Ziel der Quartärgeologie, Methoden zu ersinnen, mit deren Hilfe die eiszeitlichen Halte erfaßbar werden.

Anfangs vertrat man die Ansicht, daß nur die sogenannten Endmoränen als Äquivalente eiszeitlicher Halte anzusehen seien. Bei ihnen handelt es sich im allgemeinen um mehr oder minder deutlich entwickelte, im ganzen eisrandparallel verlaufende, gestreckte Höhenzüge aus ungeschichtetem nordischen und lokalen Moränenmaterial. Da ihre Kartierung sich auf vorgefundene Geländeformen stützt, kann man dieses Verfahren als morphologis che Methode bezeichnen.

Mit Hilfe ihrer ist es vor allem Gripp und Woldstedt in Schleswig-Holstein und im norddeutschen Tiefland, Тноме am Niederrhein gelungen, eine Reihe von Einzelvorstößen des Eises zu kartieren, die insgesamt den Eindruck reger Oszillation des Gletscherrandes während des Saale- und Weichselglazials hervorrufen. Andererseits hat die lückenhafte Entwicklung vieler Endmoränenzüge schon bald deutlich gemacht, daß Aussagen über großräumliche Zusammenhänge bei Beschränkung auf diese Methode nur schwer zu treffen sind. Entsprechend weist Gripp (1954, S. 83) darauf hin, daß „ein wenige Meter hoher flacher Rücken zur gleichen Endmoräne gehören kann wie eine benachbarte 40 oder gar $70 \mathrm{~m}$ hohe Stauchmoräne." Die morphologische Kartierung kann den ehemaligen Verlauf eines Eisrandes deshalb bestenfalls „nur aus der gesamten Gelände-Gestalt: Richtung der Rücken, Verhältnis zum Zungenbecken und zu den ... Aufhänge-Punkten der Endmoränen-Bögen “ ableiten.

Eine zweite Methode zur Kartierung eiszeitlicher Halte ist die Leitges chiebe$\mathrm{S}$ tatistik. Sie geht davon aus, daß pleistozäne Ablagerungen eines Gebietes, die aus 
ein und demselben Eisvorstoß resultieren, in ihrer Geschiebefracht gegenüber solchen vorausgegangener, nachfolgender und benachbarter Vorstöße des Inlandeises vielfach differieren. Ihre Anwendung setzt die Kenntnis der Herkunftsgebiete einer möglichst hohen Zahl von Geschieben voraus. Die methodische Anwendung dieser Kenntnisse ist besonders durch Hesemann, K. Richter und LütTIG gefördert worden.

Ein Vorteil dieser Methode liegt in der Unabhängigkeit von der Frische der Geländeformen. Andererseits differieren Eisloben verschiedener Gebiete bzw. Zeitabschnitte doch nicht stets in ihrer Geschiebefracht so deutlich, wie es zur Unterscheidung ihrer Ablagerungen wünschenswert wäre. Vor allem die Aufnahme älteren pleistozänen Materials durch jüngere Vorstöße des Inlandeises vermag die Grenzlinien der Geschiebefamilien sekundär wieder zu verwischen. Zudem ist die Häufigkeit, mit der Leitgeschiebe auftreten, beschränkt, und auch die statistische Natur der Aussage dieser Methode geht notwendig zu Lasten der Eindeutigkeit der Ergebnisse. Schließlich sind viele Leitgeschiebe nur von geringer Größe und daher fluviatil relativ leicht umzulagern.

Dennoch hat sich das leitgeschiebestatistische Verfahren vielfach bewährt und eine wertvolle Ergänzung - und manchmal auch Korrektur - der Ergebnisse der morphologischen Methode erbracht.

Besondere Schwierigkeiten stellen sich der Anwendung beider Methoden dort entgegen, wo ein bewegtes Relief Abtragung und Umlagerung der Moränen beschleunigt. Hier, das heißt praktisch im Gebiet der deutschen Mittelgebirge, soweit diese vom nordischen Inlandeis überfahren wurden, findet man auf ausgedehnten Flächen nur noch vereinzelt nordisches Material, das noch nicht umgelagert und zugleich reich an Leitgeschieben ist. Das gilt um so mehr, als es sich hier um Bildungen der Saale- und vorsaalischer Kaltzeiten handelt, so daß auch der Zeitfaktor die fortgeschrittene Abtragung der Moränen im Verhältnis zu Norddeutschland erklären hilft.

Hier nun bietet sich nach Auffassung des Verfassers als zusätzliches Hilfsmittel die Grobgesch i ebestat is t i k an. Während einer mehrjährigen, gründlichen Begehung weiter Teile der erwähnten mittelgebirgigen Landschaften habe ich zunehmend den Eindruck gewonnen, daß die Transportkraft des fließenden Wassers bei stark bewegtem Relief zwar vielfach ausgereicht hat, das gesamte feinere Material der Moränen (Endmoränen und Grundmoräne) abzutragen, nicht jedoch deren gröbste Bestandteile, die deshalb als Grobskelett auf das Liegende projiziert worden sind. Bei der Kartierung der verbliebenen Grobgeschiebe hat sich zudem gezeigt (SERAphim 1962), daß diese sehr ungleichmäßig gestreut sind. Aus einer grobgeschiebearmen Landschaft, die den Regelfall darstellt, heben sich wenige schmale Geländestreifen mit außerordentlich dichter Geschiebestreuung ab. Dies wird um so deutlicher, je radikaler die Skelettierung der Moräne selbst, aber auch die Abtragung von Nachschüttungssanden, Flottsand, Löß usw. fortgeschritten ist. Die Breite dieser Dichtestreifen beträgt, je nach Geländeform, wenige hundert Meter bis über $2 \mathrm{~km}$. Sie ist um so geringer, je steiler, jedoch um so größer, je flacher die Hangwinkel verlaufen. Sie entspricht auffällig derjenigen von morphologisch erfaßbaren Endmoränen glazialer Halte. Die Abgrenzung ist nach innen, d.h. gegen die Richtung, aus der das Eis floß, meist weniger scharf als gegen den Außenrand.

In ihrer Längserstreckung sind die Dichtestreifen in Bogen angeordnet, die sich gegen die Herkunftsrichtung des Eises, d. h. NW bis NE öffnen. Die Spannweite an den Aufhängepunkten, die mit Erhebungen im Gelände zusammenfallen, beträgt 5 bis $10 \mathrm{~km}$. Lücken im Verlauf der Dichtestreifen sind nur dort aufgetreten, wo Flußläufe mit ihrer Niederterrasse oder holozänen Alluvionen (Werre, Bega, Hase), wo eine starke Lößdecke oder wo Nachschüttungssande die tieferliegende, aber durch Bohrungen vielfach nachgewiesene Moräne bzw. deren Skelett verdecken.

Bei der Kartierung wurden nur Geschiebe oberhalb einer Mindestgröße von $40 \mathrm{~cm}$ berücksichtigt. Sie entspricht einem Erfahrungswert, der bei besonders starker Relief- 
energie, z. B. innerhalb des Teutoburger Waldes, ermittelt wurde: dort liegt der Durchmesser der nordischen Geschiebe ganz überwiegend über $40 \mathrm{~cm}$, während alle feineren Fraktionen bereits weitgehend ausgeschwemmt sind und zum Teil ganz fehlen. Andernorts, z. B. in der Herforder Liasmulde, liegt der Grenzwert zwischen den erosiv überwiegend entfernten und den von der Erosion nicht mehr erfaßbaren Fraktionen tiefer.

Innerhalb der Dichtestreifen wurde eine mittlere Streu pro qkm von 860 Blöcken oberhalb der angegebenen Größenordnung von $40 \mathrm{~cm}$ ermittelt; in Gebieten der Scharung mehrerer Dichtestreifen - z. B. an den Flanken von Bonstapel, Seel-Berg und Saal-Egge südlich von Vlotho - steigt die Streudichte auf mehr als 2300 Grobgeschiebe pro qkm. Außerhalb der Streifen werden durchschnittlich nur 8 Blöcke pro qkm erreicht.

Diese Dichtestreifen nordischer Grobgeschiebe werden als projizierte Grobskelette glazialer Halte verstanden! In Gebieten mit geringer Blockstreu handelt es sich demgegenüber um die Projektion von Grobgeschieben einer geringmächtigen Grundmoräne.

Die Anreicherung der Grobgeschiebe kann nicht Folge von Solifluktion sein. Bei Hanglage eines Dichtestreifens wurde stets beobachtet, daß nicht nur ober-, sondern auch unterhalb des Streifens nur wenige Grobgeschiebe lagen. Einwirkungen von Solifluktion wurden nur auf tonigem Untergrund festgestellt, während Sandstein und Kalk — wohl auf Grund ihrer Wasserdurchlässigkeit - kaum Solifluktion aufkommen lassen, die Grobgeschiebe mit sich führt. Da den Tonvorkommen in der Schichtstufen- und Schichtrippenlandschaft Nordwestfalens und Südniedersachsens ohnehin bereits im Präglazial die Tallage zugewiesen war (gelegentlich auf dem Wege der Reliefumkehr), kommt hier der solifluidalen Dislokation von Grobgeschieben kaum Bedeutung zu.

Eine oberflächenhafte Anreicherung von Grobgeschieben vermag allerdings dort einzutreten, wo Grundmoräne in außergewöhnlicher Mächtigkeit zur Ablagerung gelangt ist. In Vertiefungen des präsaalezeitlichen Reliefs sind Mächtigkeiten der Grundmoräne bis zu $10 \mathrm{~m}$ in der Herforder Liasmulde, z. B. bei Braunenbruch unweit Detmold, tatsächlich angetroffen worden. Jedoch handelt es sich bei diesen Ausnahmeerscheinungen gerade um solche Vorkommen von Grundmoräne, die wegen ihres Bezuges zum Relief auch heute noch vor Abtragung und damit Skelettierung geschützt sind. Lediglich anläßlich ihrer Ausbeute durch Ziegeleien wird der Geschiebegehalt ersichtlich.

Abwegig wäre der Gedanke, die kartierten Dichtestreifen seien erst durch den Einfluß des Menschen, wenn auch unabsichtlich, verursacht. Sie existieren vielmehr trotz der Veränderungen, die der Mensch in der Landschaft geschaffen hat.

Was die Grobgeschiebestatistik zu leisten vermag, ergibt sich nicht zuletzt aus dem Vergleich mit den Ergebnissen der anderen Methoden. Freilich schließen sich im allgemeinen morphologische und grobgeschiebestatistische Methode gegenseitig aus, da die Projektion der Geschiebe im Tiefland nicht hinreichend stattfindet, im Mittelgebirge jedoch die Moränen oft so weit abgetragen sind, daß sie morphologisch nicht mehr hinreichend in Erscheinung treten. Gerade in der räumlichen Ausschließlichkeit beider Methoden liegt ja die Hoffnung begründet, daß zu den „klassischen“ Gebieten der Pleistozänforschung im niederdeutschen Raum neue Arbeitsgebiete treten.

Für den in Abb. 1 dargestellten Raum südöstlich von Bielefeld ist über drei Jahrzehnte die Auffassung B̈̈rTLING's maßgeblich gewesen, nach der das Inlandeis nach dem Rückzug aus dem Münsterland "noch einmal in Westfalen in einen Gleichgewichtszustand“ kam, der „zur Aufschüttung einer bedeutenden Endmoräne führte, die auf und zwischen den südlichsten Kuppen und Kämmen des Teutoburger Waldes verläuft“ (BÄRTLING 1920, S. 11). Allerdings finden sich die von B̈̈RTLING zum Beweis angeführten "geschiebereichen Sandberge" nur in dem nordwestlich von Bielefeld gelegenen Abschnitt des Teutoburger Waldes zwischen Lengerich und Halle i. Westfalen. In dem südöstlich von Bielefeld gelegenen Abschnitt fehlen derartige, morphologisch erfaßbare Geländeformen; dennoch 


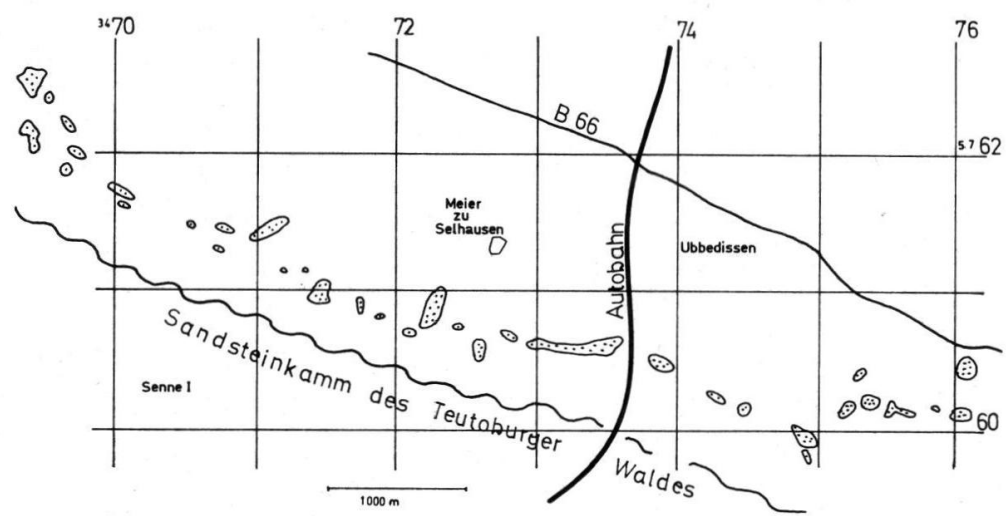

Abb. 1. Der Lämershagener Bogen des Osning-Halts in der Topographischen Karte (nach Bl. Brackwede der Karte $1: 25000$ ). Punktierte Flächen = gehäuftes Auftreten nordischer Grobgeschiebe (z. T. auf Grund von Auskünften rekonstruiert).

meint BärtLING, „daß der Eisrand auch hier lange mit den südlichsten Kämmen des Gebirges zusammenfiel. “ Keller (1952 u. a.) hat im übrigen später nachgewiesen, daß auch die zwischen Lengerich und Halle vor der Münsterlandseite der Pässe liegenden, von BÄRTLING als Reste der Osning-Endmoräne (Osning = Teutoburger Wald) gedeuteten geschiebereichen Sandberge fluviatile Bildungen verschiedenen Typs, aber keine Endmoränen sind.

Bis in die Gegenwart bedeutsam ist jedoch die Tatsache geblieben, auf die auch BärTLING hinweist, daß ein deutlicher „Gegensatz der ... Grundmoränenlandschaft nördlich des Gebirges zu den ... Heidesandflächen auf der Südseite" besteht. Dies ist es denn auch, was BärTLING ermutigt, den Verlauf des Eisrandes auch südöstlich von Bielefeld noch auf der Linie des Teutoburger Waldes zu suchen. BÄRTLING rekonstruiert hier den Verlauf der vermuteten Stillstandslage des Eises jedoch offenbar nicht mehr morphologisch, sondern unter Zugrundelegung der Reihenfolge der Glieder von glazialen Serien!

Tatsächlich ist der Unterschied zwischen den pleistozänen Deckschichten der Herforder Liasmulde im Norden und des Senneabschnittes der Münsterschen Kreidebucht im Süden so markant, daß die Annahme der Existenz einer Stillstandslage des Eises am Teutoburger Wald - wenigstens im Senneabschnitt - naheliegt (vgl. u. a. Woldstedt 1950, S. 174).

Ganz offenbar wird die Theorie von der Existenz eines solchen Haltes durch die Anwendung der Grobgeschiebestatistik bestens gestützt. Der kartierte Saum einer bisher unbekannten, außerordentlich dichten Geschiebestreuung (vgl. Abb. 1) befindet sich genau in dem Gebiet zwischen der Sanderlandschaft der Senne und der Grundmoränenlandschaft der Herforder Liasmulde.

Wenn an der Münsterlandseite des Teutoburger Waldes nordwestlich von Bielefeld die sanderartigen Bildungen deutlich abklingen, so entspricht dem die Tatsache, daß in diesem Abschnitt des Gebirges auch die Grobgeschiebedichte gering ist. Der saaleeiszeitliche Osning-Halt hat sich offenbar nur an den südöstlich von Bielefeld gelegenen Abschnitt des Gebirges angelehnt.

Genauer: der kartierte Dichtesaum der Grobgeschiebe liegt dort nördlich des Sandsteinkammes, d. h. der höchsten und zugleich geschlossensten Schichtrippe des Gebirges. BärTLING hat sich wohl nur deshalb für die „südlichsten Kämme und Kuppen“ ausgesprochen, weil er glaubte beobachtet zu haben, daß im Abschnitt Lengerich-Halle die Endmoräne diesen Kämmen noch südlich vorgelagert sei.

Die Ergebnisse der Grobgeschiebestatistik für einen erheblich größeren Raum als den in Abb. 1 exemplarisch behandelten wurden von mir 1962 in einem Zwischenbericht ver- 
öffentlicht; der vorliegende Beitrag soll in erster Linie methodologischen Zwecken dienen, die dort nur gelegentlich sichtbar werden konnten.

Freilich ließe sich zur grobgeschiebestatistischen Methode auch manches kritisch anmerken. Hierzu gehört, daß das bloße Grobgeschiebeskelett keine Schlüsse mehr auf die Menge und innere Struktur der am eiszeitlichen Halt gebildeten Ablagerungen zuläßt. Mithin ist kein Schluß auf Satz- bzw. Stauchmöränen möglich. Kurzzeitig gebildete, lokale Stauchungswälle mit nur wenig nordischem Material würden von der Grobgeschiebestatistik später wohl gänzlich übersehen.

Bezeichnend ist die Armut der Grobskelette an Leitgeschieben. Dazu trägt neben der petrographisch bedingten primär geringen Größe vieler kristalliner Leitgeschiebe auch die oft bis zwei Meter hinabreichende Verwitterung der Moräne bei. Deshalb sind die freiliegenden Grobskelette der glazialen Halte völlig frei von kalkigen und auch arm an anderen Sedimentärgeschieben von diagnostischem Wert. Auch die meisten kristallien $\mathrm{Ge}-$ schiebe tragen eine starke Verwitterungsrinde, die ihre Auswertung im Rahmen der Leitgeschiebestatistik erschwert.

Vielleicht wäre die Frage der Diskussion wert, ob eine Beziehung zwischen der Dauer eines Haltes und der Menge der Geschiebe besteht. Persönlich halte ich dies durchaus für möglich, wenngleich dabei auch andere Faktoren noch eine Rolle spielen könnten. Die Anreicherung von außerordentlich zahlreichen Grobgeschieben in schmalen Säumen könnte vielleicht ein Hinweis auf diejenigen Vorstöße des Inlandeises sein, die von längerer Dauer waren und deshalb den kurzzeitigen als Phase oder Staffel bereits übergeordnet sind.

Kritisch ist noch anzumerken, daß im Wesen der reinen Grobgeschiebestatistik liegt, daß sie wohl Kenntnis vom Verlauf von glazialen Halten, nicht aber von deren zeitlicher Reihenfolge vermittelt. Die Beantwortung dieser Frage ergibt sich vornehmlich aus der Koordination von stratigraphischen und leitgeschiebestatistischen Ergebnissen. Überhaupt bedeutet die Anwendung der einen Methode nicht die Vernachlässigung der Möglichkeiten anderer, die an bestimmten Stellen auch im Bereich des Mittelgebirges durchaus gute Ergebnisse liefern können.

Für die praktische Feldarbeit mit der grobgeschiebestatistischen Methode dienen die folgenden Hinweise:

Die Kartierung der in Frage kommenden nordischen Blöcke erfolgt zweckmäßig in Vergrößerungen der Topographischen Karten 1:25000 auf den Maßstab 1:10000, die als Arbeitskarten im Gelände dienen. Soweit möglich, erfolgt Eintragung von Einzelblöcken in diese Karten. Größere Ansammlungen, wie sie im Bereich der Grobskelette von eiszeitlichen Halten aufzutreten pflegen, sollte man in der Arbeitskarte kennzeichnen und in einem Protokoll mit laufenden Nummern näher beschreiben (Stückzahl, Situation usw.). Der Verlauf der Halte wird spätestens bei der Übertragung der im Protokoll geführten Ansammlungen in Karten des Maßstabes 1:25000 oder 1:50000 ersichtlich.

Anthropogener Einfluß läßt sich bei der Kartierung nicht ganz ausschalten. Die Verfrachtung von Grobgeschieben aus den Äckern an nahegelene Waldränder, in Bacheinschnitte oder zum Hof des Eigners kann allenthalben beobachtet werden. Die Ränder der Dichtestreifen werden dadurch jedoch im allgemeinen nicht verwischt, da sich der Transport der schweren Blöcke schwierig gestaltet und der „Einzugsbereich“ der Höfe gering ist. Dennoch sollte man, besonders bei Anreicherung von Blöcken auf Höfen, in Siedlungen usw. außerhalb der Dichtestreifen, die Herkunft der Geschiebe zu klären versuchen. Von weither stammen manchmal Zwecksteine an Wegecken, Einfahrten, in Fundamenten, an Gedenkstätten usw. Man sollte sie an Ort und Stelle mitkartieren, es sei denn, ihre Herkunft ließe sich noch eindeutig klären, so daß sie in ihrem Ursprungsgebiet vermerkt werden müßten. Das ist aber zumeist nicht mehr der Fall. Nie sind sie übrigens so zahlreich, 
daß dadurch das durchschnittliche Mengenverhältnis vom Grobskelett glazialer Halte zu dem von Grundmoräne (im nordwestfälisch-lippischen Bergland und der östlichen Hälfte der Herforder Liasmulde 860:8 pro qkm) wesentlich verschoben werden könnte.

Lediglich Friedhöfe sind bei der Erfassung der Grobgeschiebe grundsätzlich ausgespart worden.

\section{Literatur}

BäRTLING, R.: Die Endmoränen der Hauptvereisung zwischen Teutoburger Wald und Rheinischem Schiefergebirge. Z. deutsch. geol. Ges. 72, 1920.

Burre, O.: Ein Endmoränenbogen bei Herford und Bünde i. Westf. Jb. Preuß. Geol. L.-A., 306-311, 1923.

GrIPP, K.: Glacialmorphologie und geologische Kartierung, zugleich eine Deutung der Oberflächenformen Ost-Holsteins. Z. deutsch. geol. Ges., 99, 190-205, 1949. - - Die Entstehung der Landschaft Ost-Schleswigs vom Dänischen Wohld bis Alsen. Meyniana, Verh. Geol. Inst. Kiel, 2, 1954.

Grupe, O.: Die Kamesbildungen des Weserberglandes. Jb. Preuß. Geol. L.-A., 51, 350-370, 1930. Herrmann, R.: Eisrandbildungen und Fließerden am Nordrand des Leine-Berglandes. Geol. Jb., \%6, 309-320, 1958.

Hesemann, J.: Statistische Geschiebeuntersuchungen. Z. f. Geschiebeforschung, 6, 159-162, 1930. - Quantitative Geschiebeuntersuchungen im norddeutschen Diluvium. Jb. Preuß. Geol. L.-A., 51, 714-758, 1931. - - Über die Bedeutung von Korngröße, Verwítterung und Art der Ablagerung für die Geschiebeführung. Z. f. Geschiebeforschung, 9, 1-6, 1933. - Ergebnisse und Aussichten einiger Methoden zur Feststellung der Verteilung kristalliner Leitgeschiebe. Jb. Preuß. Geol. L.-A., 55, 1-27, 1934. - - Elster- und Saaleeiszeit in Westfalen und anschließendem Rheinland nach ihrer Geschiebeführung. N. Jb. Geol. Pal., Mh., 49-54, 1956.

HUскE, K.: Über horizontale und vertikale Geschiebeverbreitung. Z. f. Geschiebeforschung, 2, 1926.

JASPERSEN, P.: Uber die Ursachen der Richtungsänderungen der Eisströme im nordeuropäischen Inlandeis. N. Jb. Miner., Mh., 541-553, 1957.

Keller, G.: Neue Ergebnisse der Quartärgeologie Westfalens, XII. Zur Frage der Osning-Endmoräne bei Iburg. N. Jb. Geol. Pal., 71-79, 1952.

LANG, H. D.: Das Endmoränengebiet der Mellendorfer und Brelinger Berge. Ber. Naturhist. Ges., 107, 21-30, Hannover 1963.

LÜтtIG, G.: Alt- und mittelpleistozäne Eisrandlagen zwischen Harz und Weser. Geol. Jb., yo, 43-125, 1954. - - Eiszeit - Stadium - Phase - Staffel. Eine nomenklatorische Betrachtung. Geol. Jb., 76, 235-260, 1958. - - Methodische Fragen der Geschiebeforschung. Geol. Jb., 75, 361-418, 1958.

Naumann, E.: Erläuterungen zur Geologischen Karte von Preußen usw., Blatt Rinteln. Berlin 1922.

RichteR, K.: Über den morphologischen Nachweis großer Schwankungen des Eisrandes. Eiszeitalter u. Gegenwart, 1, 65-69, 1951. - - Geschiebegrenzen und Eisrandlagen in Niedersachsen. Geol. Jb., 76, 223-234, 1958.

Seraphim, E. Th.: Glaziale Halte im südlichen unteren Weserbergland. Zwischenbericht. Spieker, H. 12, 46-80, Münster 1962. - - Das Physiotop-Gefüge des Bielefelder Osnings. Inaug.Diss., Münster 1964.

Tноме, K. N.: Eisvorstoß und Flußregime an Niederrhein und Zuider See im Jungpleistozän. Fortschr. Geol. Rheinld. u. Westf., 4, 197-246, 1959.

WahnschafFe, Fr.: Die Ursachen der Oberflächengestaltung des norddeutschen Flachlandes. Forsch. Dtsch. Landes- u. Volkskde., 6, 103 ff., 1891 (3. Aufl. 1909).

Wennberg, G.: Uber den Geschiebetransport im Inlandeis. Schr. naturwiss. Ver. Schlesw.-Holst., 25, 1951.

Woldstedt, P.: Über Endmoränen in der südlichen Lüneburger Heide. Abh. Naturwiss. Ver. Bremen, 31, 326-246, 1939. - - Das Eiszeitalter. Grundlinie einer Geologie des Quartärs. 1. Band: Die allgemeinen Erscheinungen des Quartärs. 3. Aufl., 1961.

Manuskr. eingeg. 24. 8. 1965.

Anschrift des Verf.: Dr. Ernst Th. Seraphim, 4816 Sennestadt, Westallee 7. 